\title{
Learning Molecular Representations for Thermochemistry Prediction of Cyclic Hydrocarbons and Oxygenates
}

Maarten R. Dobbelaere ${ }^{1}$, Pieter P. Plehiers ${ }^{1}$, Ruben Van de Vijver ${ }^{1}$, Christian V. Stevens ${ }^{2}$,

Kevin M. Van Geem ${ }^{1, *}$

${ }^{1}$ Laboratory for Chemical Technology, Department of Materials, Textiles and Chemical Engineering, Ghent University, Technologiepark 125, 9052 Gent, Belgium

${ }^{2}$ SynBioC Research Group, Department of Green Chemistry and Technology, Ghent University, Coupure Links 653, 9000 Gent, Belgium

* Corresponding author: Kevin.VanGeem@UGent.be, Technologiepark 125, 9052 Gent, Belgium

\section{Supporting Information}




\section{S1. Database and Predictions}

The complete database and the predictions are available in ML4Thermo_results.xlsx. Please cite this paper when using data from this database.

\section{S2. GauL HDAD Algorithm}

The algorithm is available on https://github.com/mrodobbe/GauL-HDAD. Please refer to this paper when publishing results with GauL HDAD. 
S3. Gaussian Mixture Models

The $\mathrm{CC}$ and $\mathrm{CO}$ range are split into smaller ranges with new labels. They are given in Table S1.

Table S1: Labels of smaller range histograms of CC and CO

\begin{tabular}{|c|c|c|c|}
\hline $\begin{array}{c}\text { Histogram } \\
\text { Label }\end{array}$ & Bond & $\begin{array}{c}\text { Lower } \\
\text { Value [Å] }\end{array}$ & $\begin{array}{c}\text { Upper } \\
\text { Value }[\AA ̊]\end{array}$ \\
\hline C1 & $\mathrm{CC}$ & 0 & 1.25 \\
\hline $\mathrm{C} 2$ & $\mathrm{CC}$ & 1.25 & 1.31 \\
\hline C3 & $\mathrm{CC}$ & 1.31 & 1.36 \\
\hline C4 & $\mathrm{CC}$ & 1.36 & 1.425 \\
\hline C5 & $\mathrm{CC}$ & 1.425 & 1.468 \\
\hline C6 & $\mathrm{CC}$ & 1.468 & 1.8 \\
\hline C7 & $\mathrm{CC}$ & 1.8 & 2.32 \\
\hline C8 & $\mathrm{CC}$ & 2.32 & 2.7 \\
\hline C9 & $\mathrm{CC}$ & 2.7 & 2.95 \\
\hline CX & $\mathrm{CC}$ & 2.95 & 4.1 \\
\hline CY & $\mathrm{CC}$ & 4.1 & $+\infty$ \\
\hline 01 & $\mathrm{CO}$ & 0 & 1.18 \\
\hline $\mathrm{O2}$ & $\mathrm{CO}$ & 1.18 & 1.25 \\
\hline $\mathbf{O 3}$ & $\mathrm{CO}$ & 1.25 & 1.4 \\
\hline 04 & $\mathrm{CO}$ & 1.4 & 2.0 \\
\hline 05 & $\mathrm{CO}$ & 2.0 & 2.27 \\
\hline 06 & $\mathrm{CO}$ & 2.27 & 2.5 \\
\hline $\mathbf{0 7}$ & $\mathrm{CO}$ & 2.5 & 3.9 \\
\hline 08 & $\mathrm{CO}$ & 3.9 & $+\infty$ \\
\hline
\end{tabular}


All histograms of distances, angles and dihedrals and the corresponding Gaussian mixture models are given below:
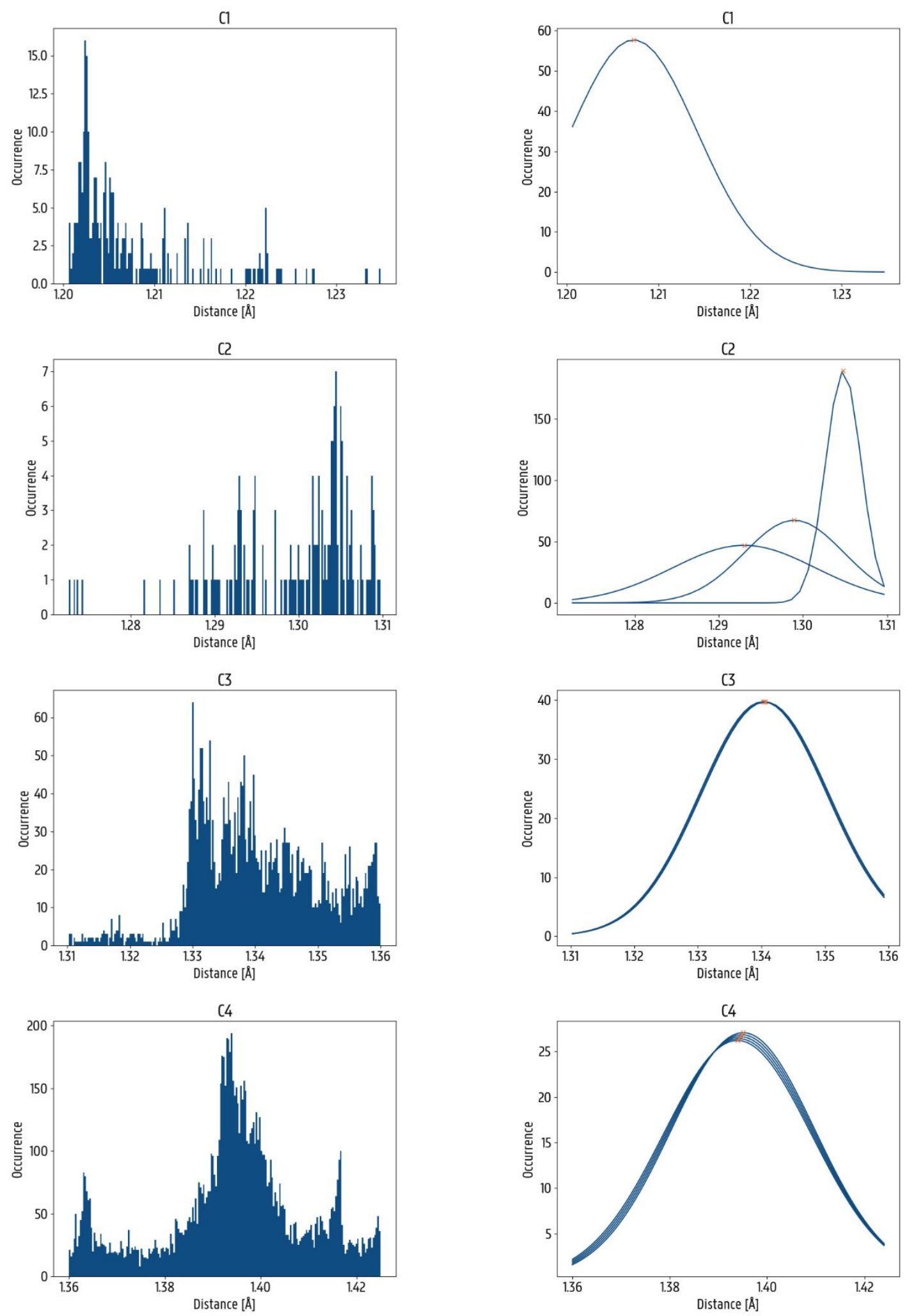

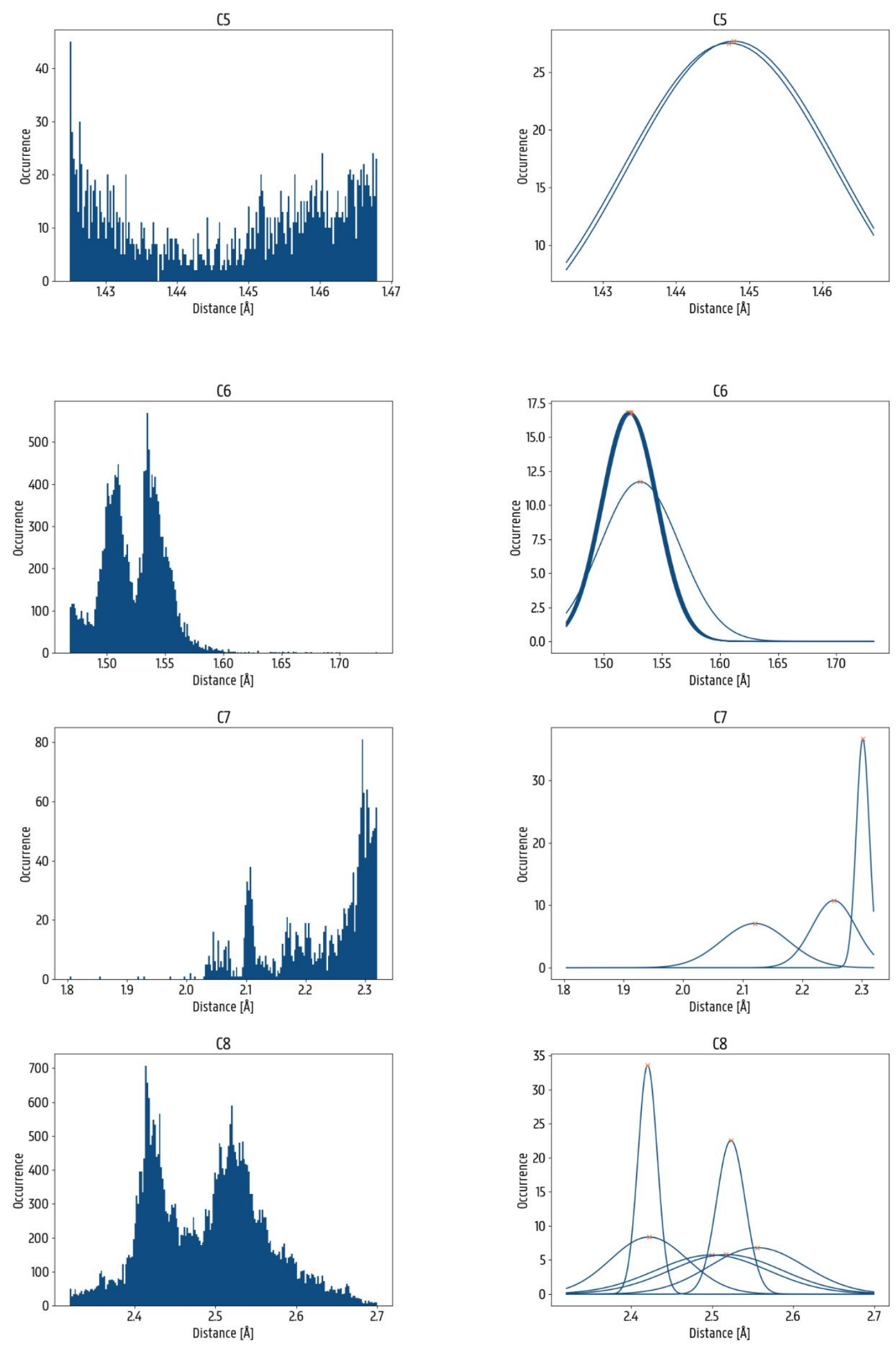

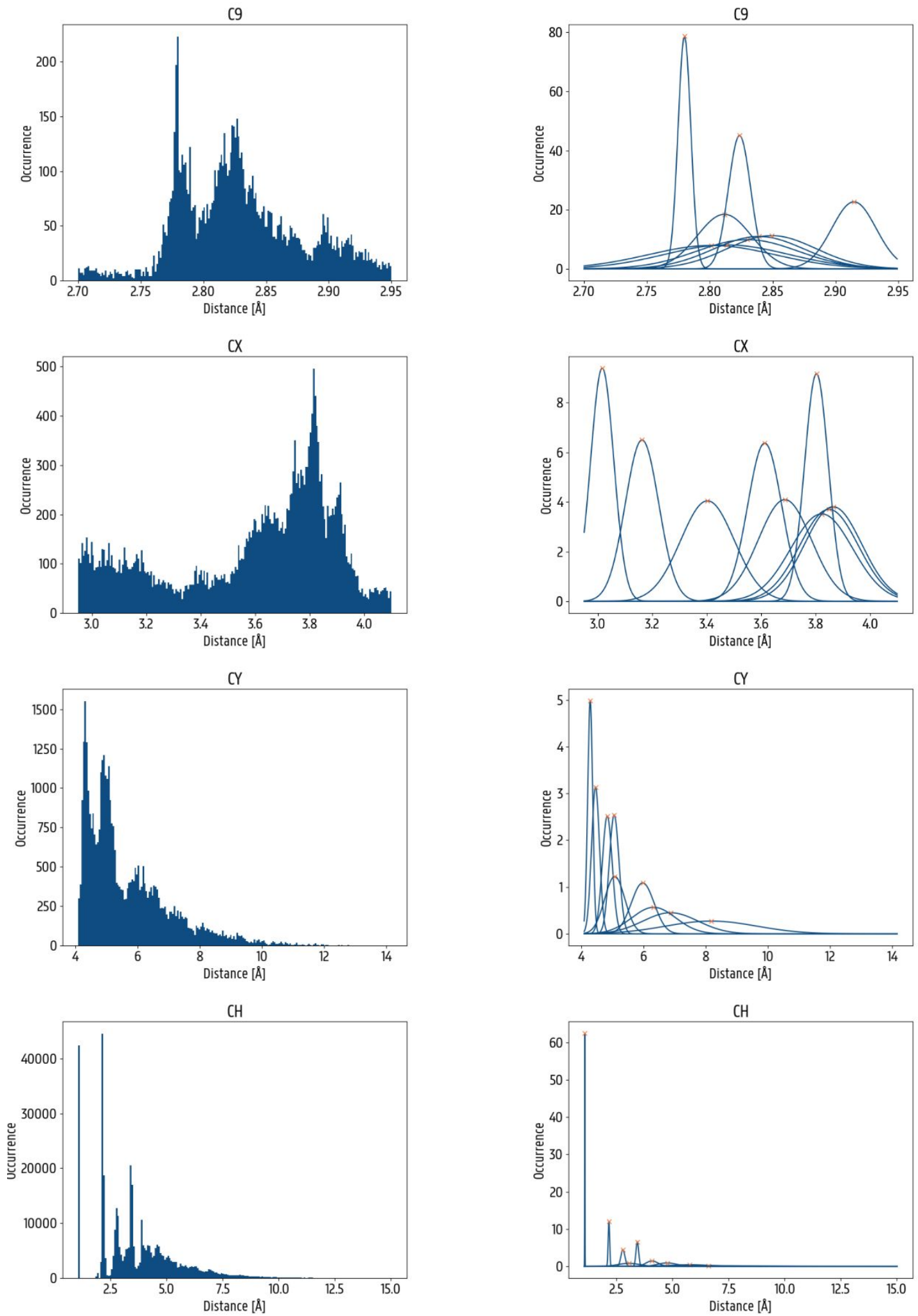

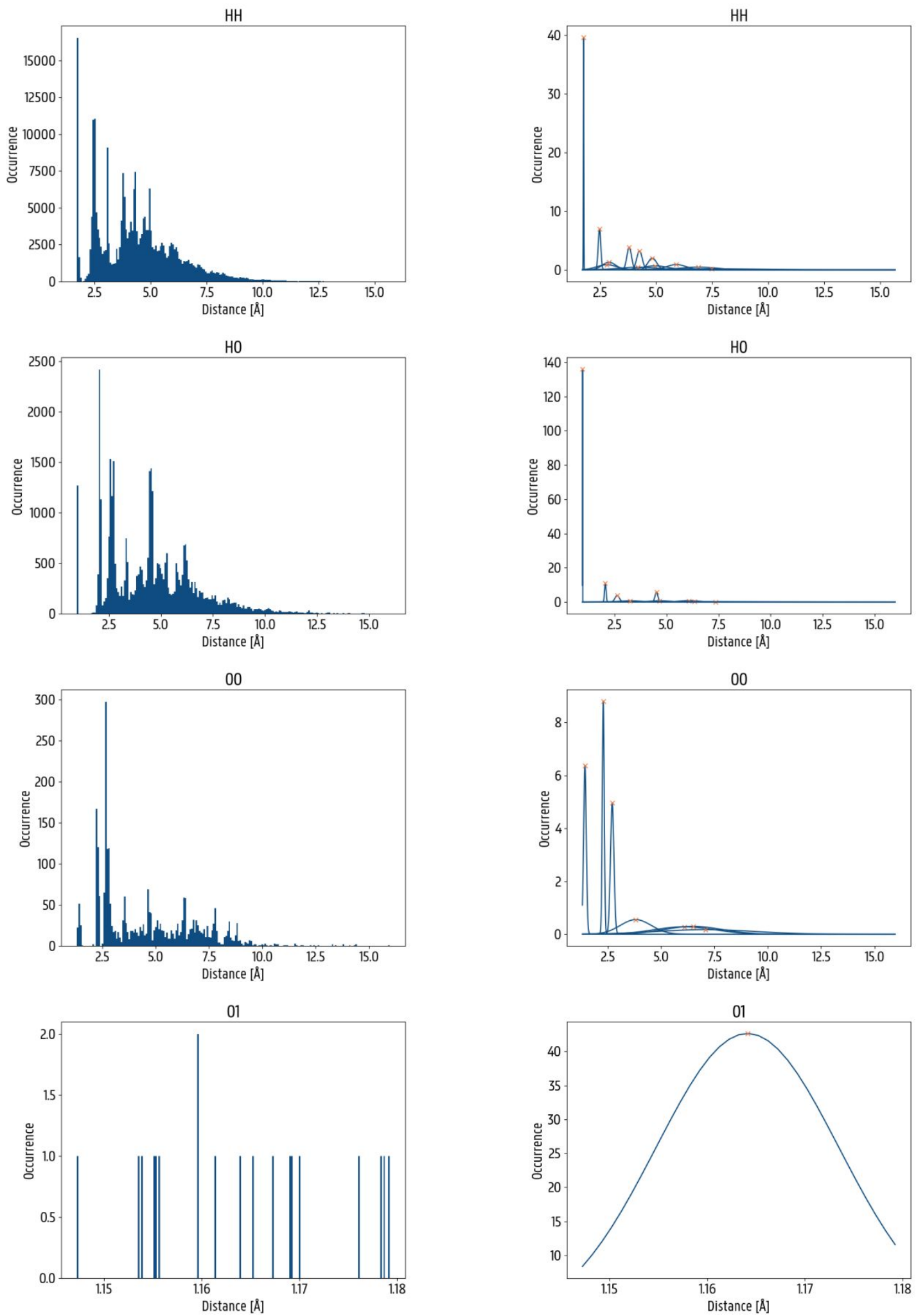

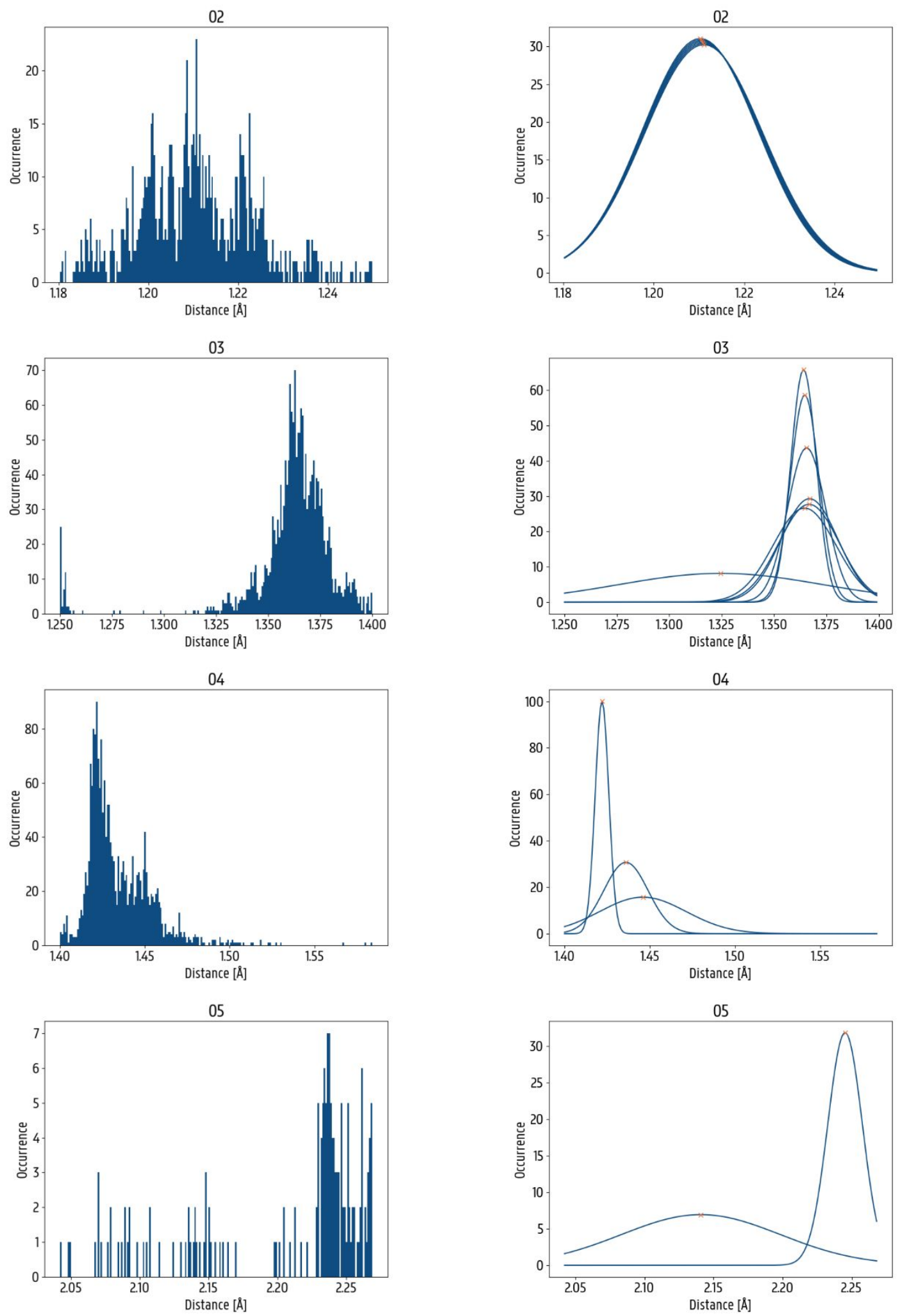

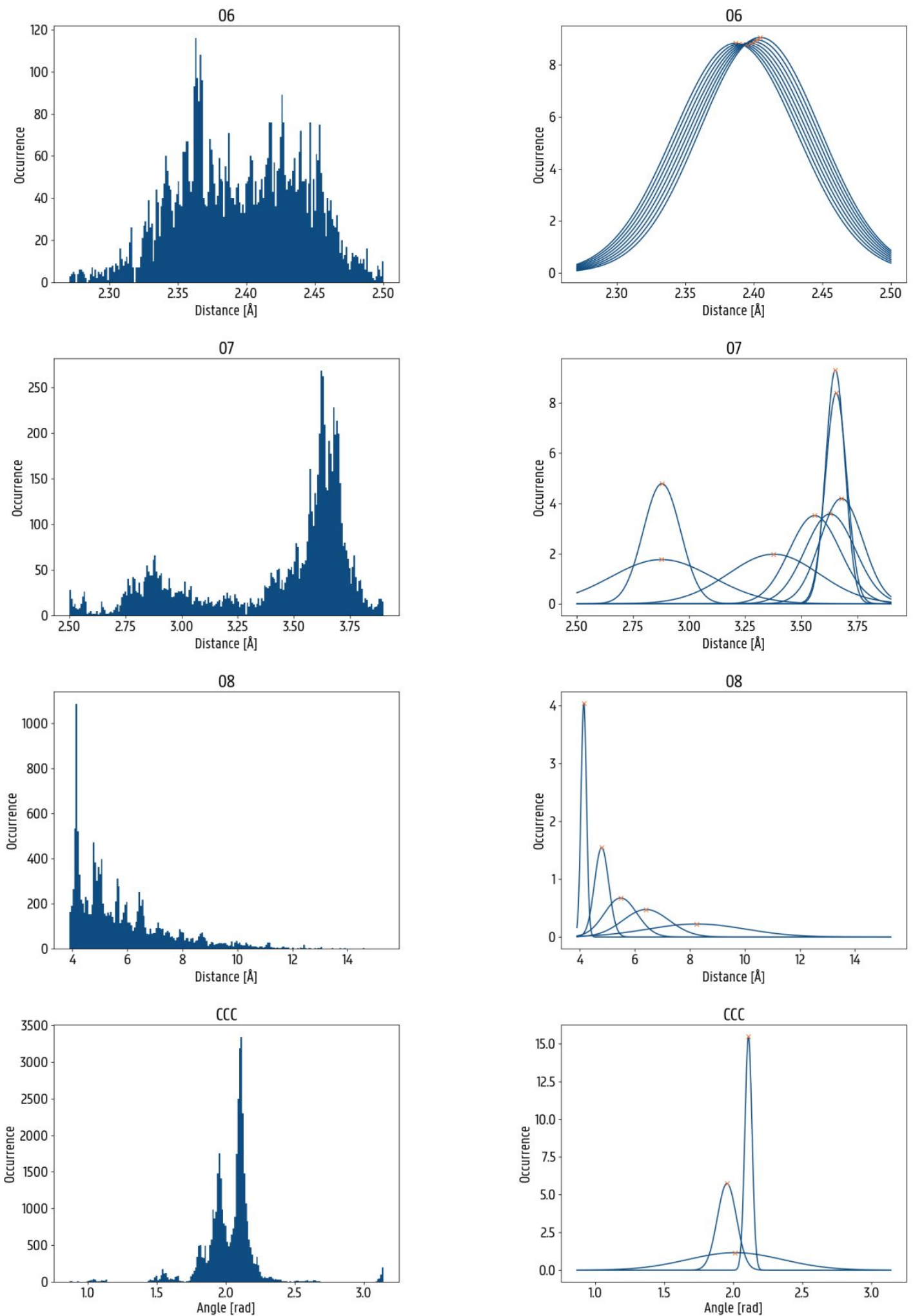

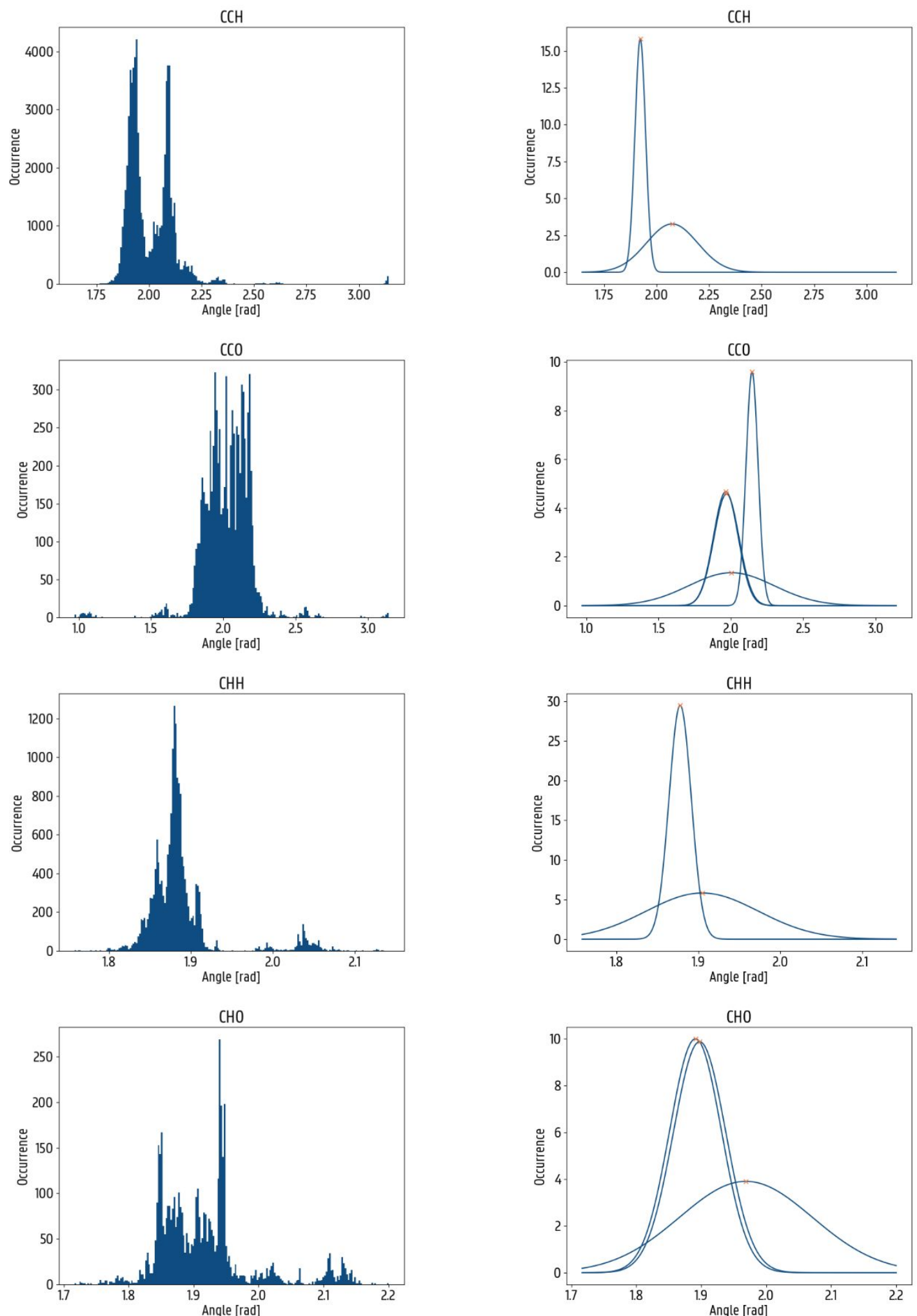

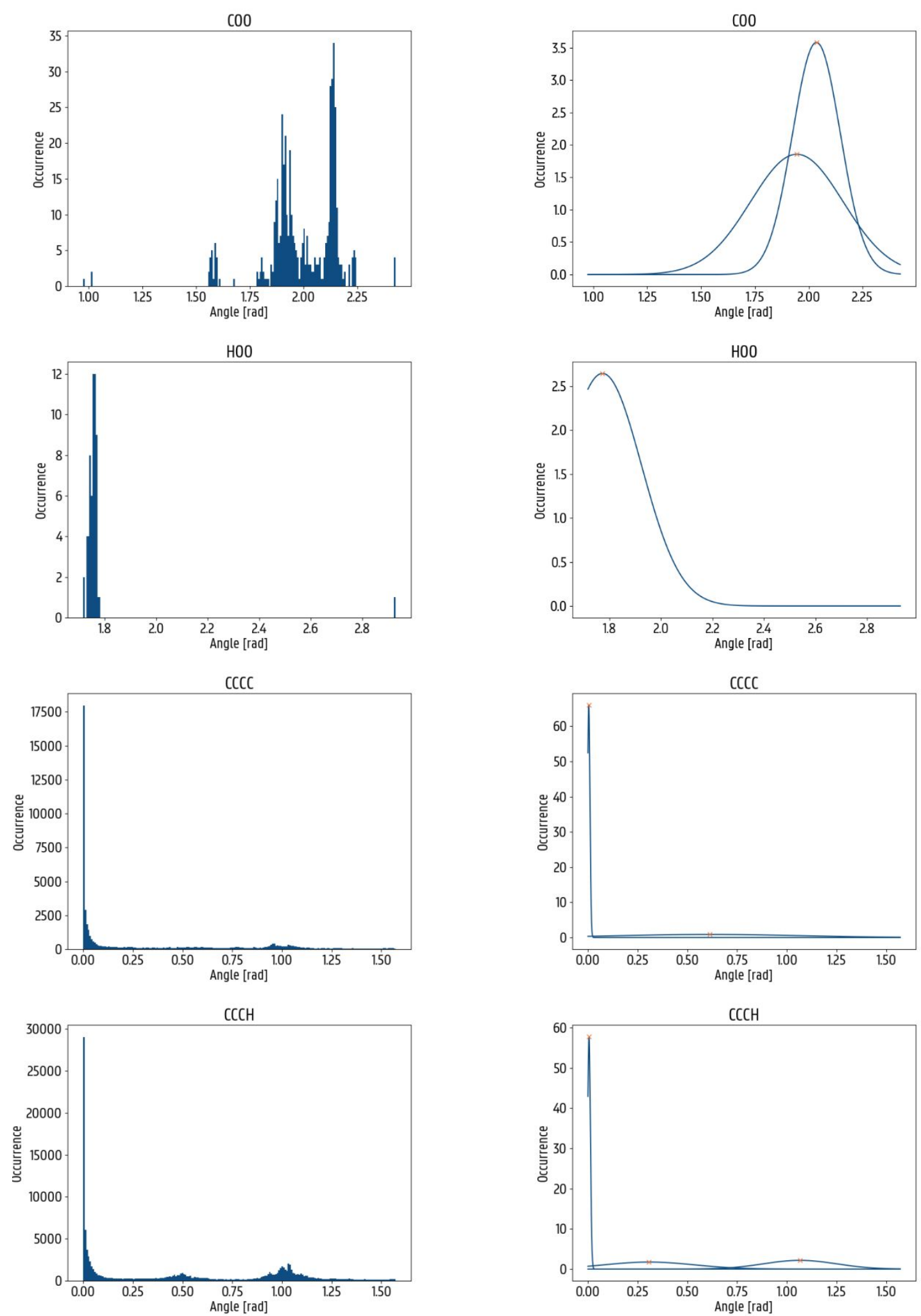

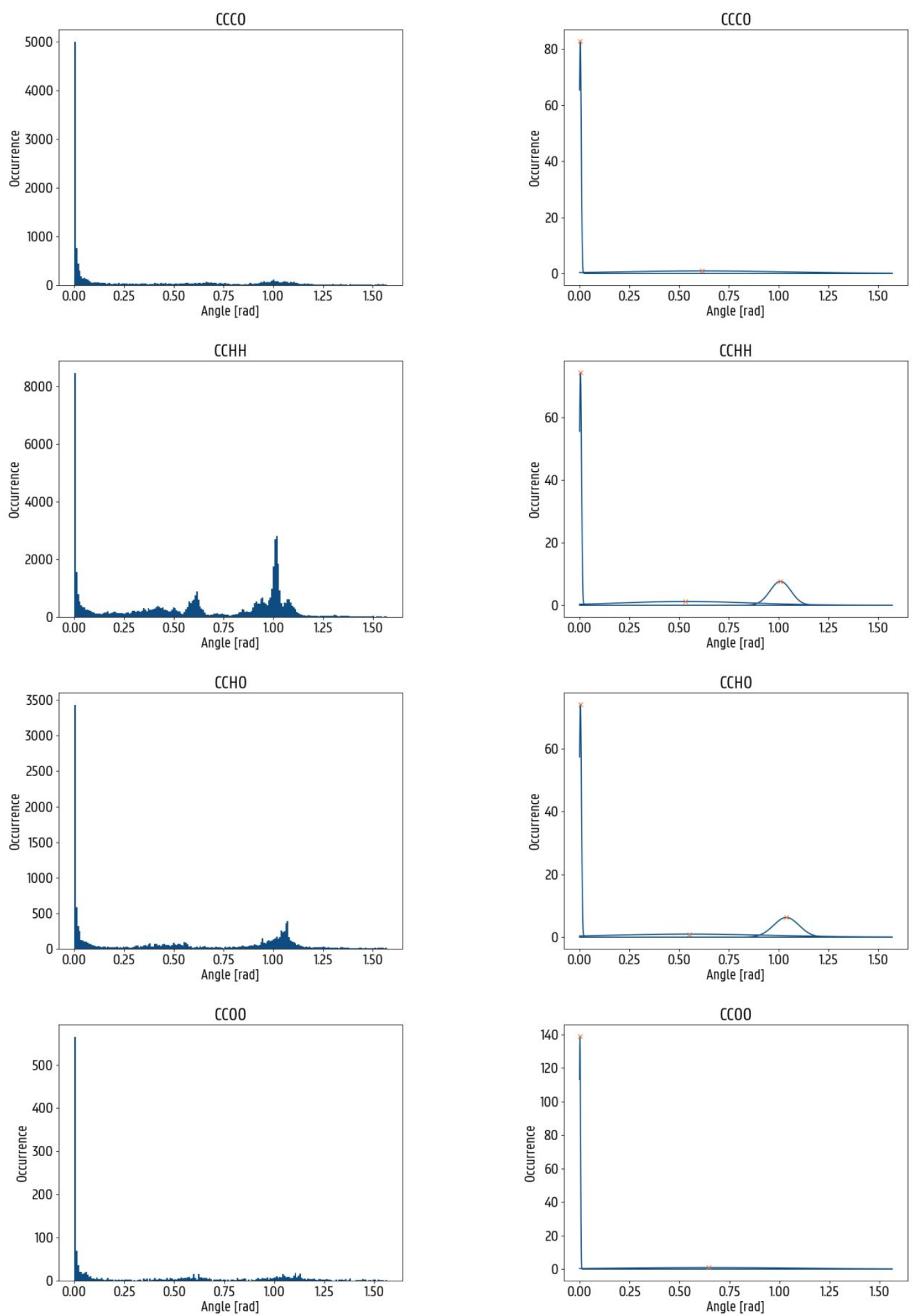

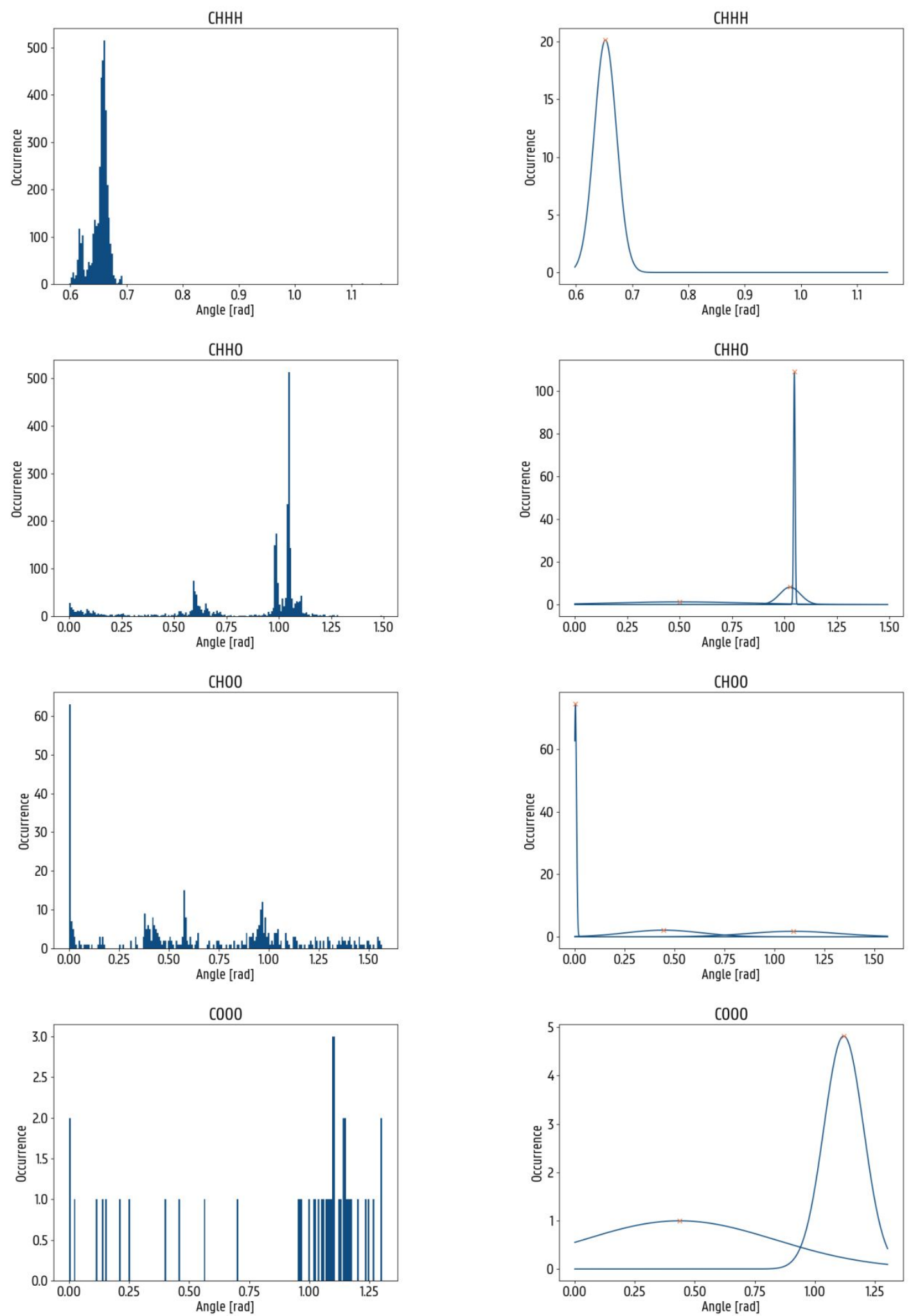


\section{S4. Hyperparameters}

The hyperparameters (number of layers, individual layer size and activation function) were optimized using the Hyperband algorithm [1] in Keras-tuner [2]. All models have 5 hidden layers and use Leaky ReLU activation functions between hidden layers and a linear activation function for the output. The hidden layer sizes are listed in Table S2.

Before using the optimizer, it was found that using 5 hidden layers works better than using another number of hidden layers. ReLU, Leaky ReLU and swish activation functions were considered in the optimizer.

Table S2: Optimized architectures for each property. Hidden layer sizes for different models

\begin{tabular}{c|ccc|c|c|} 
& & Lignin QM & & QM9 & KAUST \\
$\begin{array}{c}\text { Hidden } \\
\text { Layer }\end{array}$ & H298 & S298 & Cp & H298 & H298 \\
$\mathbf{1}$ & 860 & 400 & 200 & 1600 & 400 \\
$\mathbf{2}$ & 460 & 100 & 500 & 1000 & 200 \\
$\mathbf{3}$ & 50 & 40 & 30 & 40 & 20 \\
$\mathbf{4}$ & 560 & 800 & 600 & 200 & 550 \\
$\mathbf{5}$ & 140 & 900 & 900 & 200 & 600 \\
\end{tabular}




\section{S5. References}

1. Li, L., Jamieson, K., DeSalvo, G. et al., Hyperband: a novel bandit-based approach to hyperparameter optimization. J. Mach. Learn. Res., 2017. 18(1): p. 6765-6816.

2. O'Malley, T., Bursztein, E., Long, J. et al. Keras Tuner. 2019 - Available from: https://keras-team.github.io/keras-tuner/. 